\title{
Eine neue Seite im OP-Journal: Tipps und Tricks für den Alltag im OP!
}

$\square$ Dankward Höntzsch, Florian Gebhard

Das richtige und harmonische Operieren ist der Grundpfeiler für unser Fach Orthopädie und Unfallchirurgie. Neben den allgemeinen Richtlinien, Operationslehren und Schulen sowie Gebrauchsanweisungen sind wir auf Tipps und Tricks angewiesen. Dies sammelt jeder für sich oder schaut sie sich ab.

Gerade das OP-Journal ist geeignet, dass wir diese Tipps und Tricks untereinander austauschen. Jeder von Ihnen vom OPPflegepersonal und von den Ärzten hat sicher diesen oder jenen kleinen Tipp und Trick zur Verbesserung und Harmonisierung beim Operieren.

Dies gilt auch für die Instrumententische und Instrumentenbearbeitung im Hintergrund.

Wir wollen hier eine neue Plattform ins Leben rufen.

In jedem Heft sollen eine oder zwei Seiten mit Tipps und Tricks erscheinen. Jeder Vorschlag sollte mit Text und Bildern auf nur einer Seite dargestellt werden.
Zum Start und als Anregung sollen noch einmal Tipps und Tricks von Dankward Höntzsch gezeigt werden.

Bitte senden Sie Ihre Tipps und Tricks ein, damit dies ein lebendiger Austausch wird und wir voneinander lernen können. Bitte an: op-journal@thieme.de oder Georg Thieme Verlag KG, OP-Journal, z. Hd. Frau Stickel, Rüdigerstraße 14, 70469 Stuttgart.

Dankward Höntzsch

Florian Gebhard 